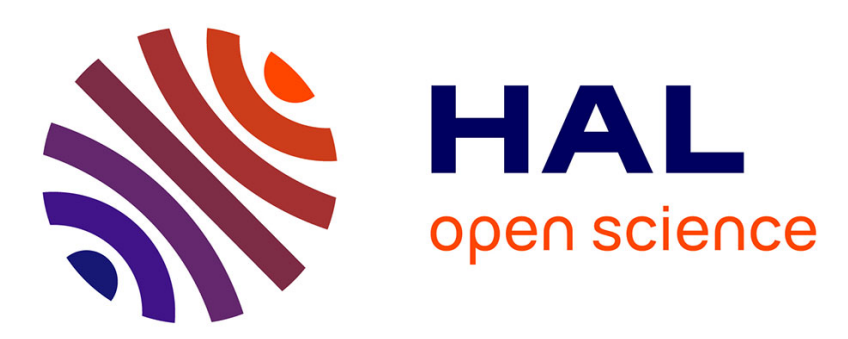

\title{
Nanostructuration effect on the mechanical properties of PMMA toughened by a triblock acrylate copolymer using multilayer coextrusion
}

Juan-Sebastian Montana, Guillaume Miquelard-Garnier, Emmanuel Richaud, Sebastien Roland

\section{To cite this version:}

Juan-Sebastian Montana, Guillaume Miquelard-Garnier, Emmanuel Richaud, Sebastien Roland. Nanostructuration effect on the mechanical properties of PMMA toughened by a triblock acrylate copolymer using multilayer coextrusion. Polymer, 2018, 149, pp.124-133. 10.1016/j.polymer.2018.06.048 . hal-01900652

\section{HAL Id: hal-01900652 \\ https://hal.science/hal-01900652}

Submitted on 22 Oct 2018

HAL is a multi-disciplinary open access archive for the deposit and dissemination of scientific research documents, whether they are published or not. The documents may come from teaching and research institutions in France or abroad, or from public or private research centers.
L'archive ouverte pluridisciplinaire HAL, est destinée au dépôt et à la diffusion de documents scientifiques de niveau recherche, publiés ou non, émanant des établissements d'enseignement et de recherche français ou étrangers, des laboratoires publics ou privés. 


\title{
Nanostructuration effect on the mechanical properties of PMMA toughened by a triblock acrylate copolymer using multilayer coextrusion
}

\author{
Juan-Sebastián Montana, Sébastien Roland*, Emmanuel Richaud, \\ Guillaume Miquelard-Garnier* \\ PIMM, UMR 8006, ENSAM - CNRS - CNAM, HESAM Université, 151 Boulevard de L'Hôpital, 75013, Paris, France
}

\section{A R T I C L E I N F O}

\section{Article history:}

Received 27 February 2018

Received in revised form

1 June 2018

Accepted 16 June 2018

Available online 18 June 2018

Keywords:

Multilayer coextrusion

Directed self-assembly

Mechanical reinforcement

\begin{abstract}
A B S T R A C T
Multilayer coextrusion was used to obtain nanolayered films of self-assembled commercial triblock copolymer poly(methyl methacrylate- $b$-butyl acrylate- $b$-methyl methacrylate) (MAM) confined by poly (methyl methacrylate) (PMMA). A double level of organization is achieved: the obtained films are made of thousands of alternating continuous nanolayers of each component, while within the nanolayers, MAM is self-assembled with a cylindrical morphology. The mechanical properties of the resulting films were compared to those of virgin PMMA and to classically extruded PMMA/MAM blends with the same compositions where no control of the nanostructure can be achieved. It is shown that significant reinforcement can be obtained with the multilayer films compared to virgin PMMA (on the order of 20 times in terms of fracture toughness) but also compared to classical blends, by a factor from 2 to 4 . Reinforcing glassy thermoplastics has been a major industrial challenge due to their usually brittle behavior in the temperature range they are used. This industrially scalable one-step process shows promises for the design of nano-laminated organic glasses with high impact resistance.
\end{abstract}

\section{Introduction}

Incorporating rubbery particles into glassy thermoplastics has long been used to toughen these usually brittle materials. A reinforcing mechanism based on the formation of crazes initiated from the rubbery particles well-dispersed in the matrix is generally admitted [1]. The most famous example of such materials is probably high impact polystyrene (HIPS) where a solution of polybutadiene (PB) rubber is dissolved in the styrene monomer so that the rubber forms discrete particles in the polystyrene (PS) during its polymerization, since the two polymers are immiscible with each other. The resulting micro-structure is the so-called and well-known salami morphology, where PS subdomains (occlusions) are dispersed in micron-sized $\mathrm{PB}$ phases randomly distributed in the PS matrix [2,3]. However, because of the size of the micro-domains, PS loses its transparency upon toughening.

Poly(methyl methacrylate) (PMMA) is another glassy polymer

\footnotetext{
* Corresponding authors.

E-mail addresses: sebastien.roland@ensam.eu (S. Roland), guillaume. miquelardgarnier@lecnam.net (G. Miquelard-Garnier).
}

whose brittleness may hinder its use for some applications. PMMA being more expensive than PS, and most of its main applications (for example glazing) still requiring high transparency [1], rubbery systems leading to smaller typical sizes of the dispersed phase have been developed to avoid the turbidity or loss of transparency observed in HIPS. The refractive index of the rubber must also be taken into account and chosen as close as possible to that of the PMMA (1.49) [4,5]. In consequence, core-shell particles with a rubbery core of a crosslinked copolymer of styrene and butylacrylate and a PMMA shell, having an outer diameter around $250 \mathrm{~nm}$ have been developed [6-9]. Several slightly different core-shell particles involving more layers have since been designed to enhance the toughening [10]. The reinforcement induced by such particles has been well characterized in the aforementioned references and others [11,12]. Especially, it has been shown that at high speeds such as those occurring when impact resistance is tested, the effect of the particles become negligible and the "reinforced" PMMA displays a brittle fracture similar to the unfilled PMMA one. In other words, the core-shell particles toughened PMMA still displays mechanical properties lower than other glassy thermoplastics such as polycarbonate (PC). 
In consequence, a new strategy has been quite recently proposed, based on the enhanced properties that may result from the miniaturization of phases in polymer blends or polymer composites, i.e. nanostructuration [13]. In the case of PMMA toughening, this strategy implies the use of triblock copolymers with two PMMA end blocks and a rubbery block which is usually an acrylate such as polyethylacrylate or more commonly polybutylacrylate (PBA), having a refraction index very close to the PMMA index (1.46). As PBA and PMMA are immiscible with each other, the blocks will phase separate, and elastomeric nanodomains having dimensions of a few tens of nanometers (depending mainly on the molar mass of the PBA block) will be dispersed in the PMMA matrix. These poly(methyl methacrylate- $b$-butyl acrylate- $b$-methyl methacrylate) (MAM) copolymers have been synthesized almost 20 years ago [14-16], their rheological and mechanical properties ("as-prepared" bulk) thoroughly described [14-17] and later on were developed industrially and commercialized, mainly by Arkema. The equilibrium morphologies of these copolymers have also been studied in details as a function of the triblock composition and taking into account the dispersity [18].

However, PMMA/MAM blends have not been to the best of our knowledge the subject of many studies yet. One may cite the work by Pinto et al. $[19,20]$ which deals with nanomembranes but discusses the PMMA/MAM (with 36 wt\% of PBA block) morphologies obtained by extrusion before foaming. A core-shell structure having dimensions of typically $20 \mathrm{~nm}$ has been observed at low MAM concentrations, oriented in the extrusion direction, with the apparition of larger aggregates at higher MAM concentrations.

Still, it is known that the block copolymer morphology can be impacted by the preparation method [21,22] and that different block copolymer morphologies will display slightly different mechanical properties $[23,24]$. In addition, it is also well known that the orientation of the block copolymer morphology, usually tuned by different processing conditions, will induce anisotropy in the mechanical properties for the Young's modulus, the elongation at break or the impact resistance [25-29].

In this study, we propose to analyze the effect of a double level of structuration in PMMA/MAM blends, inspired by laminated glass structure, on the mechanical properties of the resulting materials. For that purpose, we use an innovative one-step and industrially scalable process, multilayer coextrusion, which allows the fabrication of nanostructured blends presenting thousands of continuous alternating layers of two polymers with nanometric thicknesses [30]. The ability to use this processing tool to coextrudate block copolymers with glassy thermoplastics has been demonstrated recently [31-33] despite the fact that these materials are often extruded below their order-disorder transition temperature, i.e. display a high value of elasticity in the extrusion conditions. Korley et al. have been discussing the mechanical properties of PS reinforced by $50 \mathrm{vol} \%$ of poly(styrene- $b$-ethylene/propyleneb-styrene) (SEPS) as a function of processing parameters but have not compared them to those of PS or classically extruded PS/SEPS dry-blends [32]. A recent study in our group [34] evidenced that PMMA/MAM nanolayer blends at 90-10 wt\% displayed in the asextruded state a cylindrical morphology for a MAM presenting a lamellar equilibrium morphology. In the present article, we will then study both the effect of the blend nanolayered structure and the appearance of a well-controlled morphology for the triblock confined within PMMA layers on the mechanical properties of the material produced. For this purpose, the mechanical properties will be compared at various compositions to those of virgin PMMA and PMMA/MAM blends obtained by a classical dry-blend single-screw extrusion method, presenting a statistical distribution of the reinforcing (elongated) domains and an uncontrolled morphology of the triblock copolymer.

\section{Materials and methods}

\subsection{Materials}

Poly(methyl methacrylate-b-butyl acrylate-b-methyl methacrylate) (MAM, PMMA-PBA-PMMA) lamellar triblock copolymer was

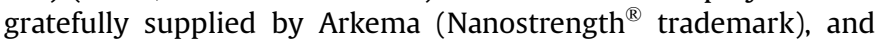
used as received without further purification. Average molecular weights $M_{w}=92.5 \mathrm{~kg} / \mathrm{mol}, M_{n}=43.2 \mathrm{~kg} / \mathrm{mol}$ and dispersity $\bigoplus_{\mathrm{M}}=$ 2.14 were previously determined [34], along with the triblock composition (23-54-23 wt\% i.e. a volume fraction of 56\% PBA and 44\% PMMA using the densities of the two polymers [18]).

Poly(methyl methacrylate) VM100 was purchased from Altuglas International and used as received as the confining polymer with the aim at reinforcing it with MAM. Its melt flow index (MFI) is 14.5 $\mathrm{g} / 10 \mathrm{~min}\left(230^{\circ} \mathrm{C} / 3.8 \mathrm{~kg}\right)$ as provided by Altuglas International. The molecular weight $M_{w}=139 \mathrm{~kg} / \mathrm{mol}$ and dispersity $\bigoplus_{M}=2.1$ were also determined previously [34]. The PMMA grade was chosen so the two polymers have similar viscosities at the temperature process $\left(225^{\circ} \mathrm{C}\right)$ and shear rates $\left(3\right.$ to $\left.20 \mathrm{~s}^{-1}\right)$.

Prior to extrusion, MAM and PMMA VM100 pellets were separately dried in a SOMOS dry air T20 eco system at $80{ }^{\circ} \mathrm{C}$ during 24 hours.

\subsection{Films preparation by nanolayer coextrusion}

Films were fabricated similarly to those studied previously $[33,34]$. Briefly, it consists in adapting a classical tri-layer coextrusion set-up by adding layer multiplying elements (LMEs), with MAM as the middle layer of the tri-layer flow and PMMA as the outer layers. A $30 \mathrm{~mm}$-diameter MAPRE and $20 \mathrm{~mm}$-diameter Scamex extruders were used respectively to set the weight composition at 70\% PMMA for 30\% MAM (wt\%) by adjusting the screw speed of each extruder to control the throughput, which is a classical blend composition for toughening applications of MAM. The whole set-up (extruders, feedblock, and LMEs) temperature was set to $225{ }^{\circ} \mathrm{C}$ i.e. below the MAM $T_{O D T}$ [33]. The theoretical number of alternating layers $(\mathrm{N})$ in the film is determined by the number of LMEs $(n)$ from the equation $N=2^{(n+1)}+1$. Depending of the targeted thickness of the confined MAM layers, 10 (2049 total layers) LMEs were used at different Draw ratios (Dr). $D r$ is defined as the ratio of the chill roll speed over extrusion flow rate at flat die and ranges from 1 to 10 (see Fig. 1 ). After the flat die, the films were drawn with a chill roll at $80^{\circ} \mathrm{C}$.

To investigate the effect of the MAM wt\% on the mechanical properties, the films detailed above were compared with multilayer films of PMMA/MAM at 90-10 wt\% with 513 total layers (8 LMEs), fabricated as previously described [34]. As will be shown below (see Table 1), the nominal layer thicknesses of 90-10 wt\% films with 513 layers are similar to those of 70-30 wt\% films with 2049 layers at a given $D r$.

Finally, pure PMMA and PMMA/MAM 90-10 wt\% and 70-30 wt $\%$ dry-blended films were obtained through simple single-screw extrusion by setting the Scamex extruder at $225^{\circ} \mathrm{C}$, to compare their mechanical properties to those of multilayer films.

For all samples, the typical total film thickness ranges from $100 \mu \mathrm{m}$ (high $\mathrm{Dr}$ ) to $1 \mathrm{~mm}$ (low $\mathrm{Dr}$ ), while the width is typically comprised between 5 and $10 \mathrm{~cm}$ and the length depends only on the fact that polymer pellets are added to the extruders.

\subsection{Atomic force microscopy (AFM)}

AFM tapping mode images were obtained by using a Multimode microscope driven by a Nanoscope V controller (Veeco) 


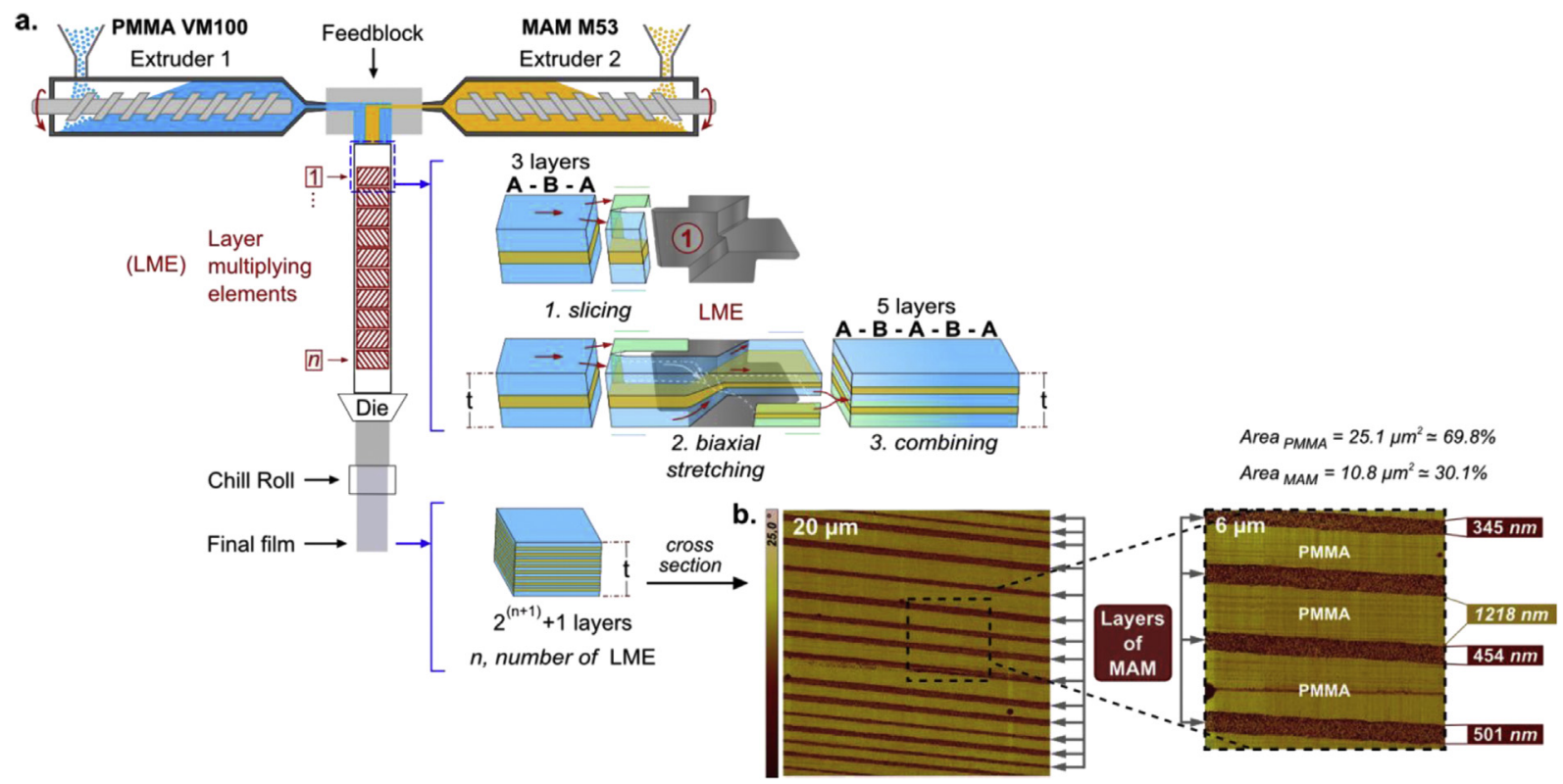

Fig. 1. (a.) Schematic of the nanolayer coextrusion setup and (b.) cross section AFM image of a coextruded film of PMMA and MAM at 70-30 wt\%.

and operated under ambient atmospheric conditions. The tips (silicon, spring constant $40 \mathrm{~N} / \mathrm{m}$, oscillation frequency ca. 300 $\mathrm{kHz}$ ) were supplied by BudgetSensors and have a curvature radius below $10 \mathrm{~nm}$. Topographic, amplitude, and phase images are acquired simultaneously, the last one allowing the microphase separation of each polymer block to be identified. Images were recorded with a resolution of $512 \times 512$ pixels and at a scan rate of $0.8 \mathrm{~Hz}$. These settings yield a pixel size of $4 \mathrm{~nm}$. In phase images, confining PMMA appears in gold and confined MAM appears in brown with golden spots corresponding to the PBA and PMMA blocks, respectively. As shown in Fig. 1, AFM is efficient to characterize the continuity of the layers in the films but does not have the resolution to study the MAM morphology within the layers.

\subsection{Transmission electron microscopy (TEM)}

The morphology of MAM copolymer within the extruded films was studied by TEM in the $x$ axis (extrusion direction, ED) and in the $y$ axis (lateral direction, LD) by using respectively the electron beam parallel and perpendicular to the ED. TEM micrographs were taken on a Zeiss $912 \Omega$ operated at an accelerated voltage of $80 \mathrm{kV}$ equipped with a side-mounted $2 k \times 2 k$ Veleta CDD camera (Olympus). Prior to the observation, the pre-faced samples of extruded films were immerged in a $1 \% \mathrm{RuO}_{4}$ solution generated in situ by combining sodium hypochlorite $(\mathrm{NaClO})$ with ruthenium (III) chloride $\left(\mathrm{RuCl}_{3} \mathrm{XH}_{2} \mathrm{O}\right)$ for 6 hours as described previously [34]. Ruthenium tetroxide stains preferentially the PBA block, giving darkest colors in the TEM analysis while PMMA appears brighter in the images. The stained samples were then sectioned at random locations perpendicular to their surface with an ultramicrotome 2088 Ultrotome V (LKB) equipped with a Diatome diamond knife at a cutting rate of $1 \mathrm{~mm} / \mathrm{s}$. Thin slices of $70-80 \mathrm{~nm}$ were obtained and placed in TEM grids for observation. At least 8 images for the $70-30 \mathrm{wt} \%$ films and 15 images for the $90-10 \mathrm{wt} \%$ films were collected from different slices.

\subsection{Small angle X-ray scattering (SAXS)}

SAXS measurements of the multilayer extruded films in the $x, y$ (extrusion and lateral directions respectively, as defined in the previous section), and $z$ (transverse direction, TD) axes were performed on the high brilliance SWING beam line at the Soleil Synchrotron facility, with a monochromator set at $12 \mathrm{keV}$ [35]. Using a CCD detector at $2 \mathrm{~m}$ from the sample, diffraction patterns were recorded for reciprocal spacing $q=4 \pi \sin (\theta) / \lambda$ varying between 0.005 and $0.4 \AA$, i.e. repetitive distances $d=2 \pi / q$ ranging from 1256 to $15 \AA$ A. By using Foxtrot software, 1D SAXS curves were obtained by circular averaging of the full $2 \mathrm{D}$ images $\left(0\right.$ to $\left.360^{\circ}\right)$, and for some non-isotropic patterns by averaging $\pm 45^{\circ}$ of the horizontal and vertical axis. 10 images were recorded for each sample and 1D curves were averaged in order to obtain 1 curve for each sample.

Blends characterizations were performed with a lab SAXS system from Xenocs. The apparatus consists of an X-ray source using a copper anode $K \alpha$ radiation, the wavelength being $1.54 \AA^{-1}$, a monochromator, a collimator, a MAR300 2D detector, and a computer equipped with the MAR300 software. Silver behenate standard reference material was used for sample-to-detector distance calibration, which was determined to be $1250 \mathrm{~mm}$. The exposure time was $15000 \mathrm{~s}$. The 2D patterns were also treated using Foxtrot software. The 2D X-ray diffraction patterns were integrated along the azimuthal direction from isotropic samples.

\subsection{Uniaxial tensile tests}

For a given set of film processing conditions, at least 6 tests were performed under controlled atmospheric conditions $\left(23^{\circ} \mathrm{C}\right.$, $\mathrm{RH}=50 \%$ ) on an Instron 5966 testing machine equipped with a 10 $\mathrm{kN}$ cell and a local strain video extensometer. Crosshead speed was set to $5 \mathrm{~mm} / \mathrm{min}$ for all tests. Dumbbell shape specimens with a standard useful zone $\left(20 \times 4 \mathrm{~mm}^{2}\right)$ according to ISO $527-2(5 \mathrm{~A})$ were prepared with a dumbbell specimen die cutter. All tests were performed up to films rupture so as to measure the elongation at break $\left(\varepsilon_{r}\right)$. 


\section{Results}

Nanolayer PMMA/MAM films were successfully extruded below MAM's $T_{O D T}$ and continuous layers were obtained with different thicknesses by varying the draw ratio. The nominal layer thickness can be determined knowing the total thickness of the film (varied by changing the draw ratio), the volume proportion of the two polymers and the number of layers (as function of the number of LMEs used) according to the equation (Equation (1)):

$t_{\text {th MAM }}=t_{\text {film }} \times \frac{v_{\text {ol }} \%_{\text {MAM }}}{n_{\text {MAM layers }}}$

Films with targeted theoretical thicknesses varying from 70 to $500 \mathrm{~nm}$ for the confined layers were prepared. The mean experimental layer thickness has been obtained by measuring a small amount of the MAM layers within the films either using AFM or TEM using the protocol described in previous works [34,36].

The results are summarized in Table 1 . The mean experimental thicknesses varied from 75 to $485 \mathrm{~nm}$, with values close to the targeted theoretical ones. The deviation between mean experimental and theoretical layer thickness may either be due to slight modifications of the composition due to small changes in the extruders throughput and/or to the sampling size (i.e. the number of layers measured in the sample compared to the total number of layers) used to determine the mean [36]. Some heterogeneity in draw direction), have been thoroughly described previously [34]. Unfortunately, the images obtained for the 70-30 wt\% films are less conclusive, due to a poorer contrast, especially in ED, that cannot be outcome by modifying the staining protocol. If PMMA lines may still be seen in LD, it is hard to distinguish the nanostructure within the layers in ED, which may either be PMMA lines (meaning lamellar structures) or PMMA dots (cylinders).

To discuss this in further details, let us now move on to the SAXS measurements.

SAXS analyses were run on the samples following the procedure described in materials and methods. The results for the multilayer films show typical anisotropic patterns in the $x, y$, and $z$ directions for relatively thick layers (Fig. 3). It appears that the patterns at for the $70-30$ wt\% films at $D r=1$ are somewhat different from what is observed in the case of $90-10 \mathrm{wt} \%$ films [34]. While the patterns for the $90-10 \mathrm{wt} \%$ films indicate a cylindrical morphology (see especially the hexagonal pattern in ED in Fig. 3), the 70-30 wt\% films patterns could correspond to a lamellar morphology as they show a transverse isotropic morphology. Nonetheless, the 70-30 wt\% films patterns at $D r=2$ (see Figure SI2 in the supplementary material section) and $\mathrm{Dr}=8$ (see Fig. 3) reflect an anisotropic morphology close to the one observed for the $90-10 \mathrm{wt} \%$ films, which could correspond to cylinders oriented in the extrusion direction. Indeed, the concentrated intensities respectively at the equatorial and at the pole for TD and LD patterns and the circular ring for ED show that linear objects are aligned in the flow direction. This effect is

Table 1

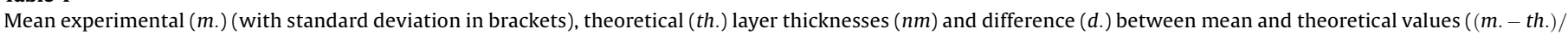
$($ th. $) \times 100)$ ) for all multilayer samples.

\begin{tabular}{|c|c|c|c|c|c|c|c|c|c|c|c|c|c|c|c|c|}
\hline \multirow[t]{2}{*}{ \% wt (PMMA/MAM) } & \multirow{2}{*}{$\frac{\mathrm{Dr}}{\mathrm{LME}}$} & \multicolumn{3}{|l|}{1} & \multicolumn{3}{|l|}{2} & \multicolumn{3}{|l|}{4} & \multicolumn{3}{|l|}{8} & \multicolumn{3}{|l|}{10} \\
\hline & & $\mathrm{m} .(\mathrm{nm})$ & th. $(\mathrm{nm})$ & $\mathrm{d}(\%)$ & m. (nm) & th. $(\mathrm{nm})$ & $\mathrm{d}(\%)$ & $\mathrm{m} .(\mathrm{nm})$ & th. $(\mathrm{nm})$ & $\mathrm{d}(\%)$ & m. (nm) & th. $(\mathrm{nm})$ & $\mathrm{d}(\%)$ & $\mathrm{m} .(\mathrm{nm})$ & th. $(\mathrm{nm})$ & $d(\%)$ \\
\hline $90 / 10$ & 8 & $\begin{array}{l}471 \\
(162)\end{array}$ & 527 & 10.7 & $\begin{array}{l}296 \\
(53)\end{array}$ & 316 & 6.4 & $\begin{array}{l}168 \\
(27)\end{array}$ & 186 & 9.2 & $\begin{array}{l}117 \\
(21)\end{array}$ & 127 & 8.1 & $\begin{array}{l}91 \\
(18)\end{array}$ & 82 & 10.9 \\
\hline $70 / 30$ & 10 & $\begin{array}{l}486 \\
(110)\end{array}$ & 513 & 5.2 & $\begin{array}{l}221 \\
(78)\end{array}$ & 300 & 26.4 & $\begin{array}{l}168 \\
(30)\end{array}$ & 158 & 6.4 & $\begin{array}{l}100 \\
(15)\end{array}$ & 105 & 5.0 & $\begin{array}{l}74 \\
(19)\end{array}$ & 70 & 5.1 \\
\hline
\end{tabular}

terms of individual layer thicknesses within the sample has also been observed, similar to what was obtained in our previous work [34], which was attributed by Korley [31] to the effects of elasticity of the BCPs extruded below $T_{O D T}$.

Structural characterization of the 70-30 wt\% PMMA/MAM multilayer and blend films was performed by TEM and SAXS measurements.

Fig. 2 shows the different representative morphologies of MAM within the extruded films at $D r=1$. Similar observations were made at higher $D r$, i.e. $D r=8$ (see Supplementary Material, Figure SI1). Single screw dry-blended films display MAM nodules at the micronscale slightly oriented in the lateral direction (LD). Some nodules, especially in the $90-10 \mathrm{wt} \%$ blends (see Fig. 2b and d), are broken, creating droplets with typical size of $100 \mathrm{~nm}$ showing higher orientation at high drawing levels. The blend structure is reminiscent of the HIPS structure with smaller typical sizes: subdomains of PMMA (occlusions) can be identified within larger elastomeric (PBA) domains dispersed within the PMMA matrix.

The expected multilayer structure of MAM and PMMA is achieved and observed in the coextruded films for both compositions. Concerning the 90-10 wt\% multilayer films, grey dots (PMMA) surrounded by darker PBA regions can be seen within the layers in ED (Fig. 2a), while PMMA lines are observed in LD (Fig. 2a'). These observations, suggesting a cylindrical morphology (more precisely PMMA cylinders within the softer PBA phase, aligned in the flow/ more pronounced when the layer thickness decreases, i.e. at high draw ratios $(D r)$.

To explain these results, let us recall that its high dispersity combined to its relatively low molecular weight makes the atequilibrium morphology of the MAM used in the study close to the lamellar-cylinder transition [34]. Shear induced by multilayer coextrusion can then favor, if sufficient enough (which may not be the case for the 70-30 wt\% films at low Dr, i.e. the thickest initial MAM layer in the tri-layer flow before the LMEs), the cylindrical morphology over the lamellar one.

1D scattering profiles were obtained by integrating SAXS patterns in the three directions. The spectra concerning the 70-30 wt\% composition in all directions and treated with a Lorentz-corrected function ( $q$ as function of $I \times q^{2}$ ) are presented in Fig. 4. Characteristic $q$ values were obtained by measuring minima of the spectra second derivative. These spectra allow observing the inter-domains behavior explained above and summarized in Table 2. Characteristic distances $\mathrm{d}_{\mathrm{E}}, \mathrm{d}_{\mathrm{L}}$, and $\mathrm{d}_{\mathrm{T}}$ (for Extrusion, Lateral, and Transverse direction respectively) were calculated using the relation $d=2 \pi / q$ and these values are given in Table 2 .

It can be observed that $d_{E}$ and $d_{L}$ follow the same trend when the layer thickness decreases: firstly, a value of $\sim 33-34 \mathrm{~nm}$ is found for thicker layers, then these inter-domains distances drop at $D r=2(\sim 30 \mathrm{~nm})$, followed by an increase at higher $D r$, reaching values close to the initial one $(\sim 31-33 \mathrm{~nm})$. However, $\mathrm{d}_{\mathrm{T}}$ values 
$\operatorname{Dr}=1$

$E D$
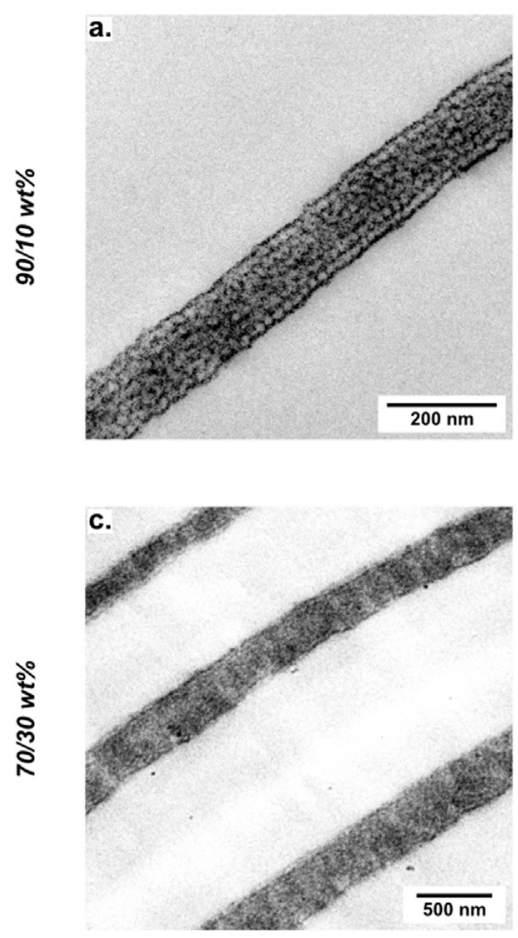

$L D$
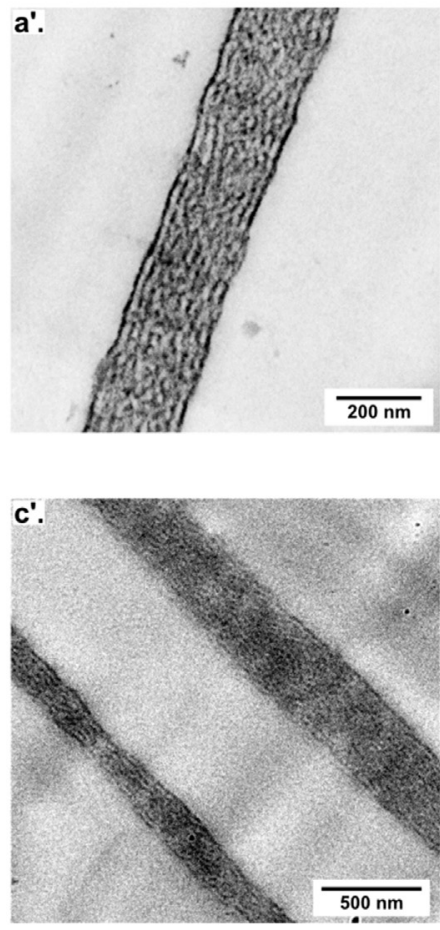

Blend
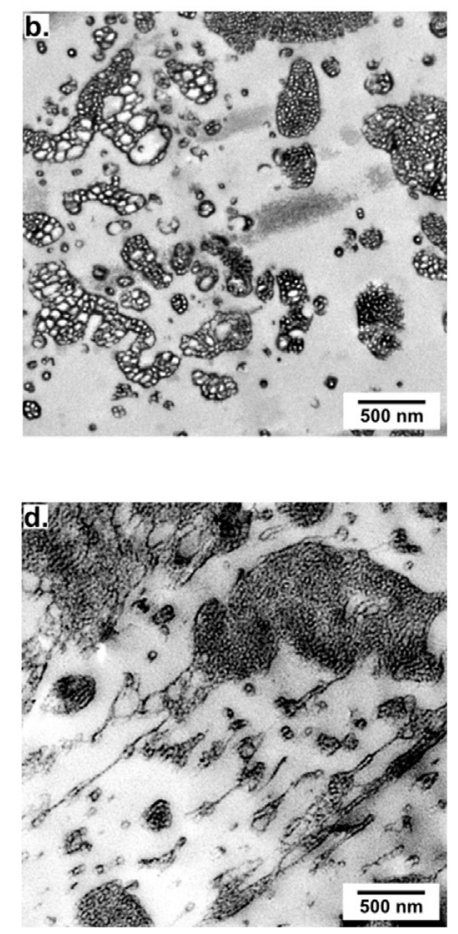

Fig. 2. Cross section TEM images of PMMA and MAM films at $D r=1$ with compositions of $90-10 \mathrm{wt} \%$ (a, a', and b) and 70-30 wt\% (c, c' and d), obtained via multilayer coextrusion (in the extrusion direction: $a$ and c; in the lateral direction: $a^{\prime}$ and c') and dry blend (in the extrusion direction: $b$ and d).

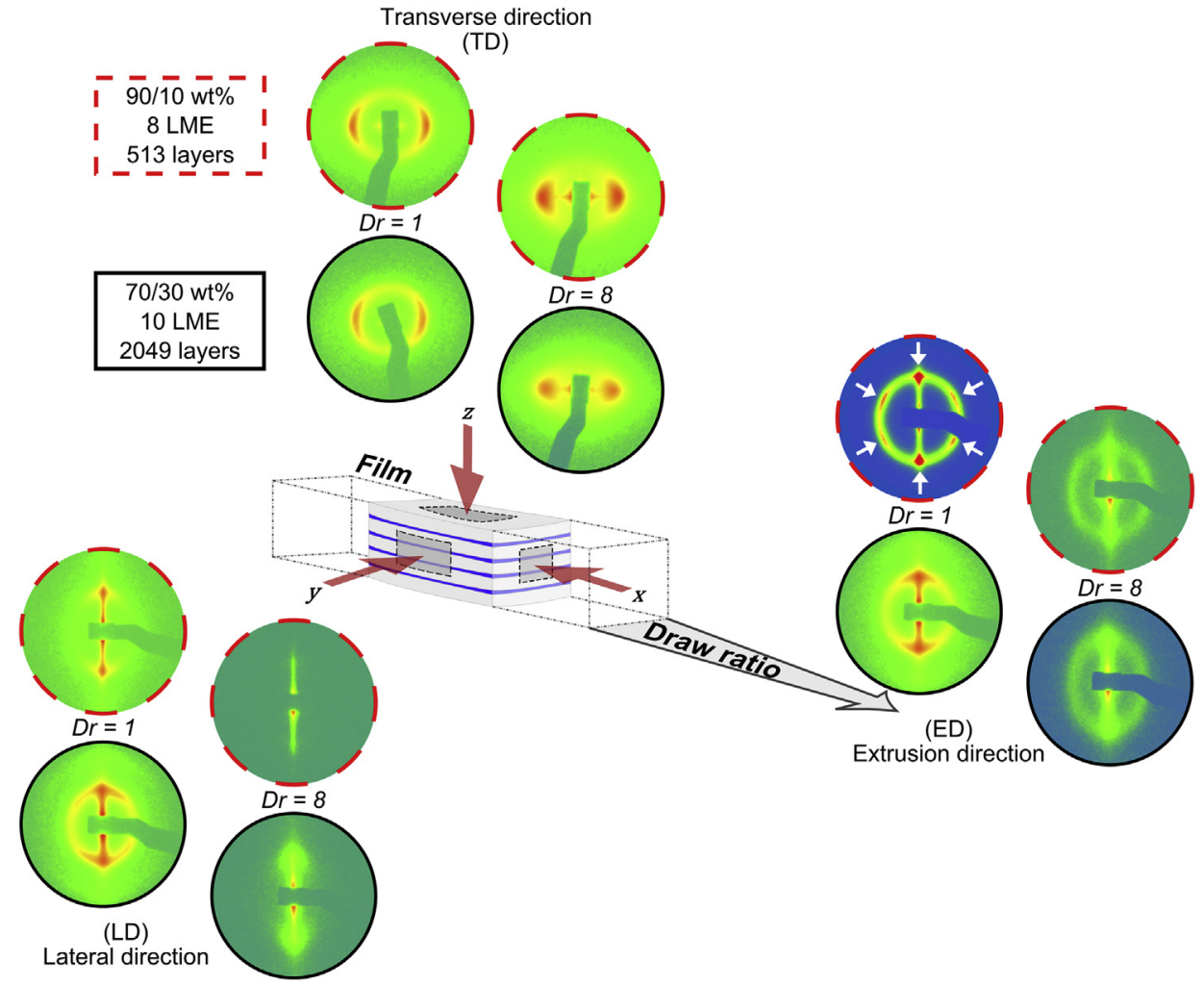

Fig. 3. SAXS patterns in $x$ (ED), $y(\mathrm{LD})$, and $z$ (TD) directions of nanolayered films with 513 layers ( $90-10 \mathrm{wt} \%$ and $8 \mathrm{~L} \mathrm{ME}$ ) and with 2049 layers ( $70-30 \mathrm{wt} \%$ and $10 \mathrm{LME}$ ) from ( $\mathrm{Dr}=$ 1) thicker to $(\mathrm{Dr}=8)$ thinner layer thicknesses. 


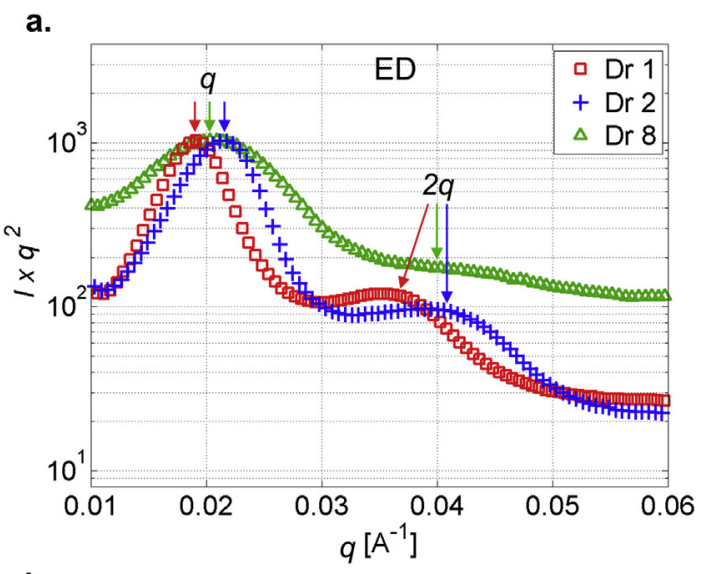

b.

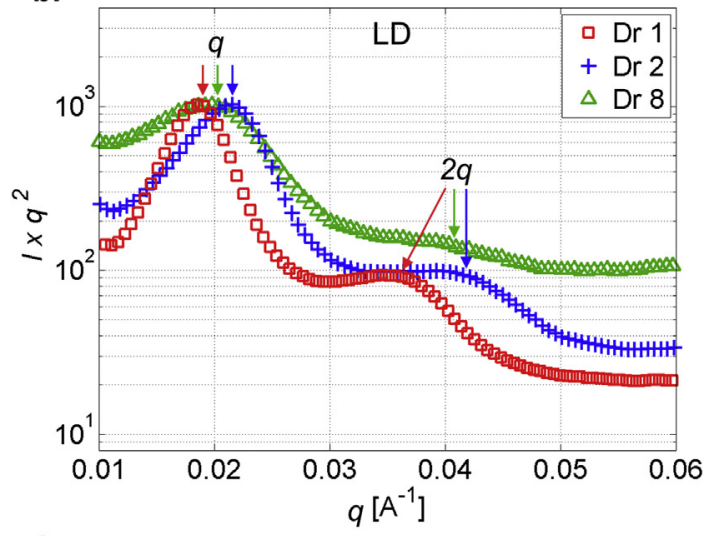

c.

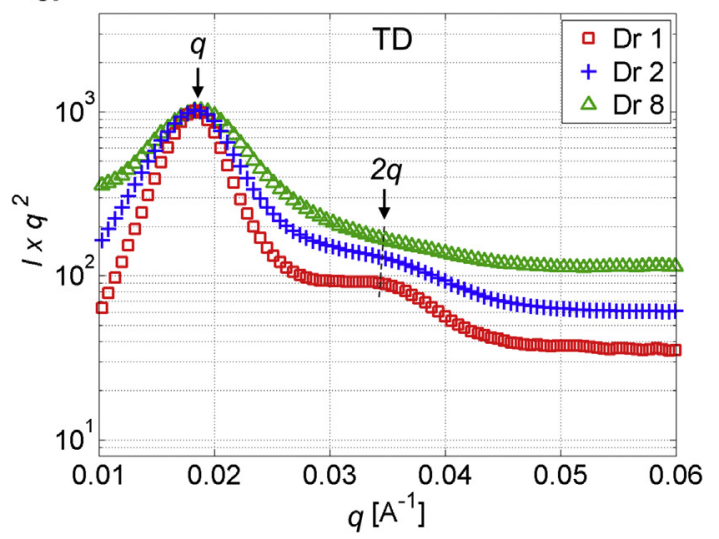

Fig. 4. Lorentz-corrected ( $I q^{2}$ as function of $q$ ) $1 \mathrm{D}$ scattering profiles in (a.) $x$ (ED), (b.) $y$ (LD), and (c.) $z$ (TD) directions of nanolayered films with 2049 layers at different layer thicknesses. The arrows point to the $q$ and 2q peak maxima.

\section{Table 2}

Distances ( $d=2 \pi / q, \mathrm{~nm})$ corresponding to the first peak from the SAXS 1D scattering profiles in $x$ (ED), $y(\mathrm{LD})$, and $z(\mathrm{TD})$ directions of nanolayered films at different layer thicknesses.

\begin{tabular}{|c|c|c|c|}
\hline $\mathrm{Dr}$ & $\mathrm{ED} \mathbf{d}_{\mathbf{E}}(\mathrm{nm})$ & LD $\mathbf{d}_{\mathbf{L}}(\mathrm{nm})$ & $\mathrm{TD} \mathbf{d}_{\mathbf{T}}(\mathrm{nm})$ \\
\hline 1 & 33.3 & 34.4 & 35.5 \\
\hline 2 & 29.7 & 29.7 & 36.7 \\
\hline 4 & 30.5 & 31.4 & 35.5 \\
\hline 8 & 31.4 & 32.3 & 36.7 \\
\hline 10 & 31.6 & 33.3 & 36.9 \\
\hline
\end{tabular}

remain almost constant at $\sim 36 \mathrm{~nm}$ whatever the $D r$ (hence the layer thicknesses). These results are similar to previous observations on the 90-10 wt\% films [34].
Assuming a perfect cylindrical order, peaks at $\sqrt{ } 3 q$ and $\sqrt{ } 7 q$ should be visible in Fig. 4. However, though the peak $2 q$ is well identified, the peaks broadness does not allow to clearly see them, except perhaps at high $\operatorname{Dr}$ for $\sqrt{ } 3 q$. This broadness is typical in such systems, and similar to what has been observed in our previous work and by other authors on both MAM and multilayer samples [18,32,34].

SAXS analyses of dry-blended films were also performed in the $x, y$, and $z$ directions showing a privileged orientation of MAM nodules in the ED and are shown in Fig. 5. Because of the pattern similarities, only the $70-30 \mathrm{wt} \%$ composition is presented. It is seen that the concentrated intensities in the TD and LD patterns respectively at the equatorial and at the pole are less pronounced than in multilayer system because of the disordered structures (see Fig. 5). Here, MAM morphology in ED is more difficult to interpret with a more or less isotropic pattern which becomes more pronounced at the poles when the film thickness decreases. Nevertheless, these patterns confirm the lack of order in MAM morphology observed by TEM micrographs. Characteristic distances between 32 and $37 \mathrm{~nm}$ can be identified depending on the observation direction and the draw ratio, similar to values obtained for the multilayer films.

The mechanical response of the different compositions for the blends has then been studied by uniaxial tensile tests. Fig. 6 shows the stress-strain curves for neat PMMA, dry blends and multilayer films in the $x$ and $y$ directions (only one representative curve of the six specimens tested is plotted). $90-10 \mathrm{wt} \%$ films (i.e. containing $5.4 \mathrm{wt} \%$ of rubbery phase) display a slight increase in the elongation at break $\left(\varepsilon_{r}\right)$ compared to neat PMMA.

However, it is needed to increase the amount of rubbery phase to really see its effect on the mechanical properties. Hence, at $70-30 \mathrm{wt} \%$ (16.1 wt\% of rubbery phase), the ductility of the resulting material is much improved over neat PMMA and 90-10 wt\% films. Moreover, at these proportions of rubbery phase, multilayer films show higher values of $\varepsilon_{r}$ than the dry blends. This behavior may be explained by well-organized structure of the multilayer films at a double level: continuous alternating submicronic layers with MAM cylinders within the confined layers.

Fig. 7a shows the Young's modulus $(E)$ for all the films studied as a function of $D r$. $E$ values of PMMA neat films remain almost constant ( $\sim 3.3$ and $\sim 3 \mathrm{GPa}$ in ED and LD respectively) whatever the draw ratio, which is not surprising (at these relatively low values of draw ratios, hardening due to strong chain orientation in the extrusion direction should not be expected). However, these films become more brittle at high $\mathrm{Dr}$ in both directions (lower values of $\varepsilon_{r}$ in Fig. 7b).

Unsurprisingly, the modulus of the films decreases with an increasing proportion of soft phase, but the differences between dry blends and multilayer films seem slight if existent. The elongation at break $\varepsilon_{r}$ is also plotted in the figure. As expected it shows a strong increase with the amount of soft block in the blend. The increase is very small for $90-10 \mathrm{wt} \%$ composition but it becomes as high as a factor of 4 over neat PMMA for 70-30 wt\% films. Large dispersity in the $\varepsilon_{r}$ values at $D r=1$ for the $70-30 \mathrm{wt} \%$ blend films may originate from the random and disordered morphology of the MAM nodules. However, for these films, a clear elongation at break improvement for multilayer films is observed over dry blends by roughly a factor of 2 , in both directions and whatever the $D r$.

The previous results can be summarized in Fig. 8 plotting the fracture toughness for the different compositions and fabrication processes studied. It was calculated by integrating the stress-strain curves of each of the six samples for every composition and $\mathrm{Dr}$ studied. It is shown that, for sufficient amount of soft phases in the 


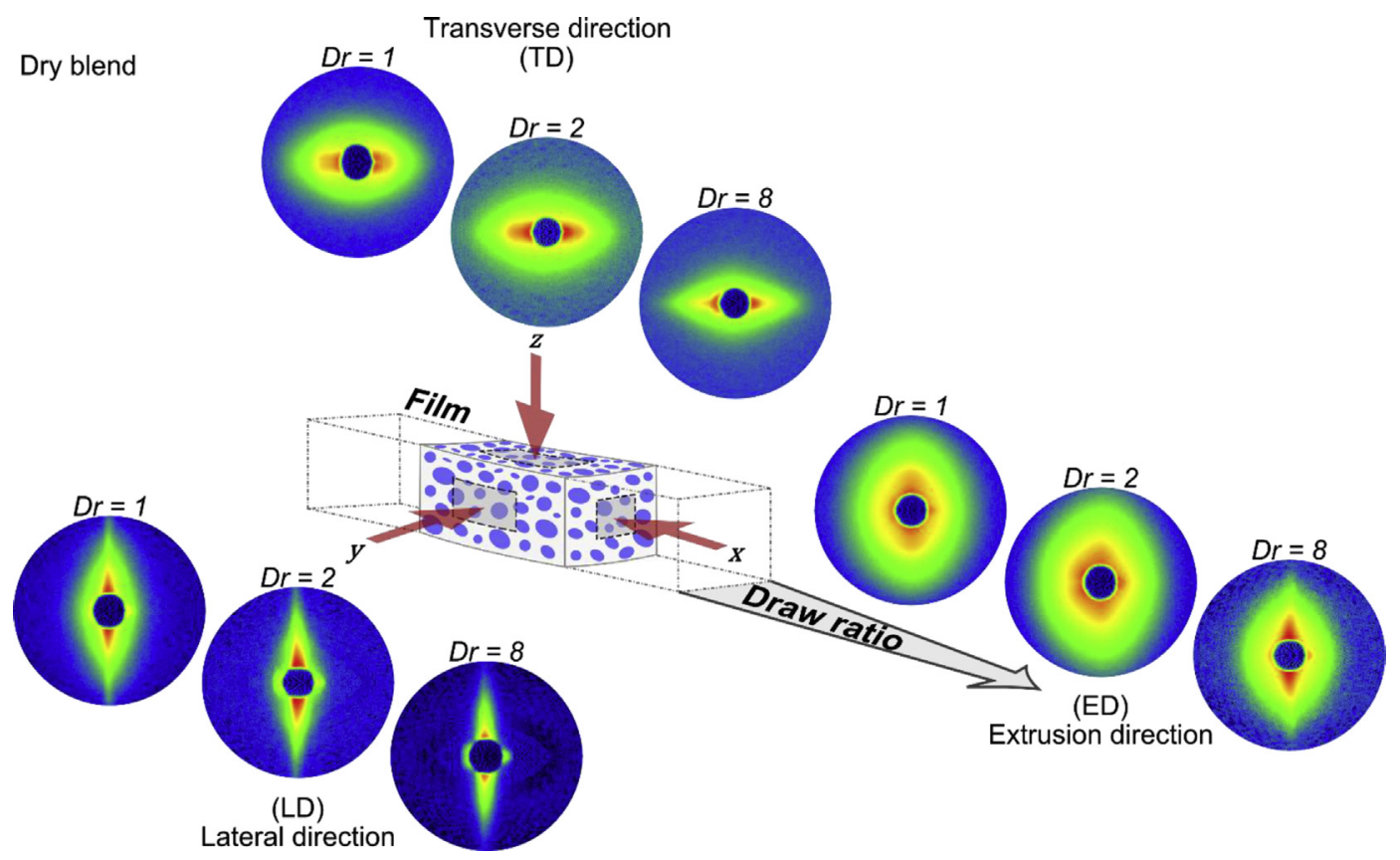

Fig. 5. SAXS patterns in $x$ (ED), $y(\mathrm{LD})$, and $z(\mathrm{TD})$ directions of dry blend films from $(D r=1)$ thicker to $(D r=8)$ thinner total film thicknesses.

a.

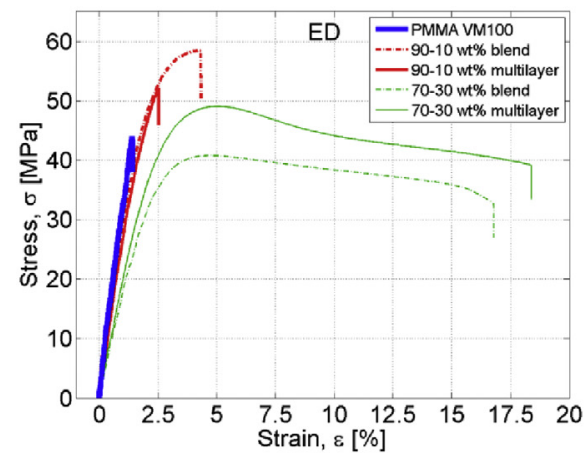

b.

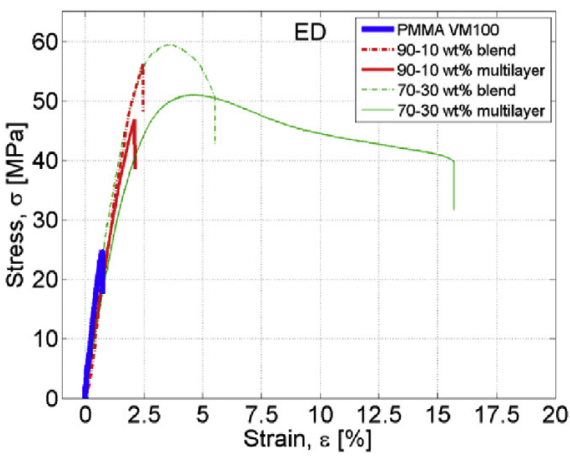

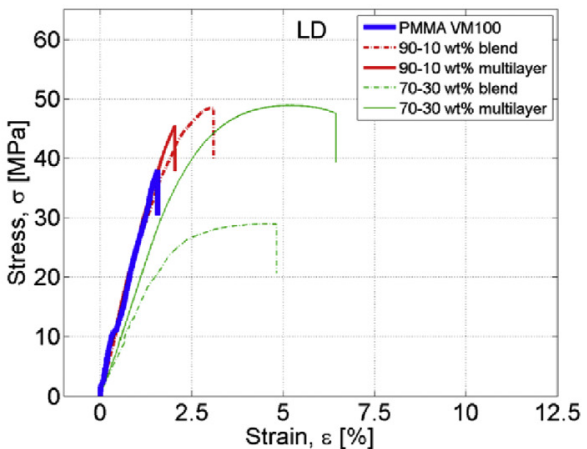

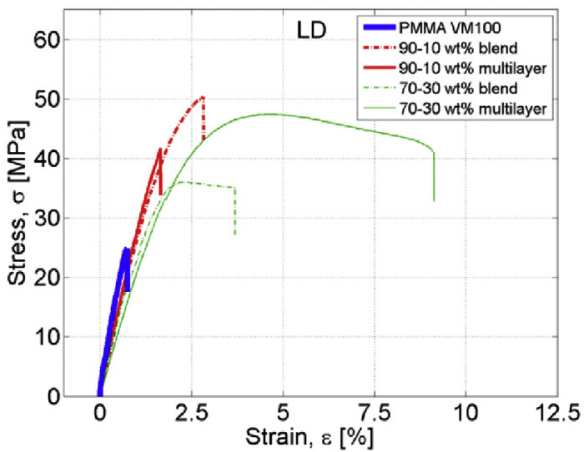

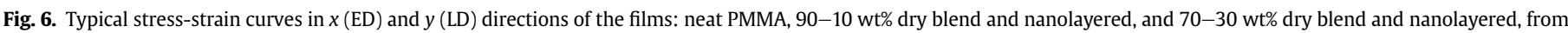
(a.) thicker to (b.) thinner total film thicknesses ( $D r=1$ and $D r=8$ respectively).

blends (i.e. for $70-30 \mathrm{wt} \%$ blend having $16.1 \mathrm{wt} \%$ of rubbery phase), the multilayer structure improves significantly (by more than a decade in the extrusion direction and in the lateral one) the fracture toughness over neat PMMA. More surprisingly the reinforcement is also more efficient than the one obtained for the dry blend at the same composition (by roughly a factor of 2 in the extrusion direction, and by a factor of 4 in the lateral one).

The obtained values for fracture toughness are similar to those found by Korley on PS/SEPS 50-50 vol\% multilayer films with a SEPS having $21 \mathrm{vol} \%$ of styrene, which means roughly $40 \mathrm{wt} \%$ of rubbery 
a.
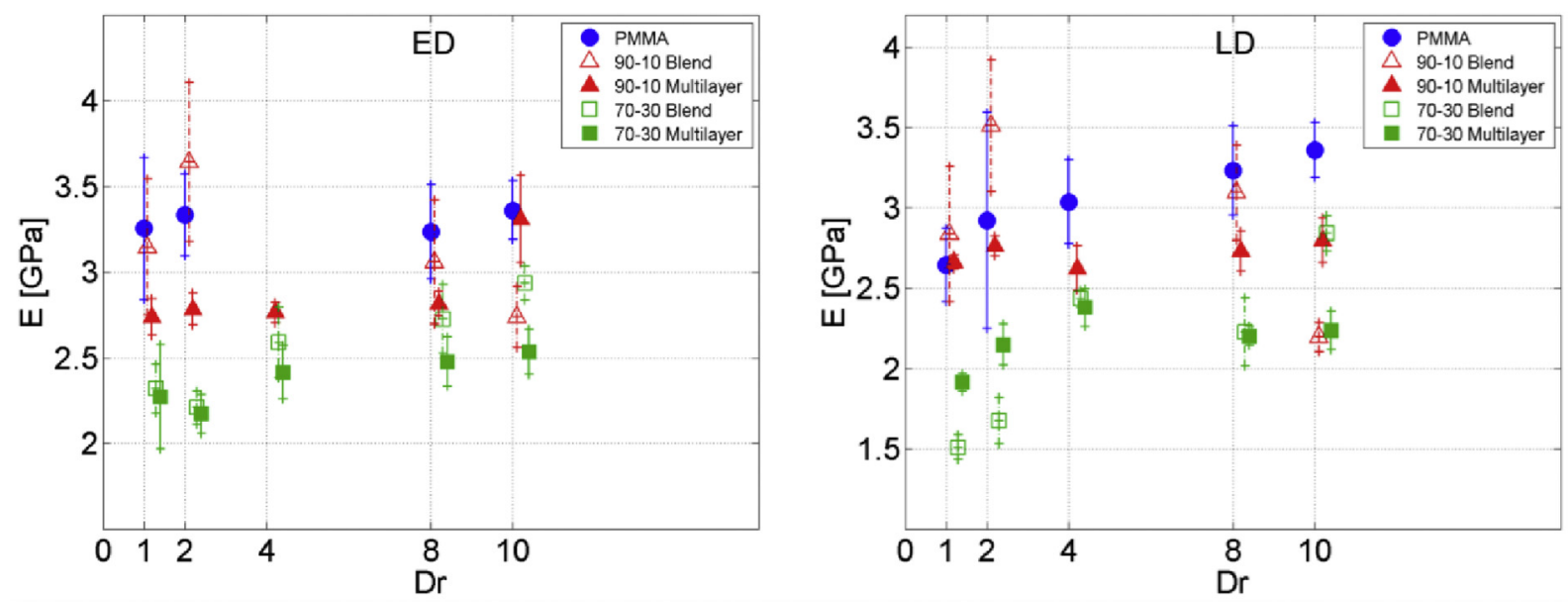

b.
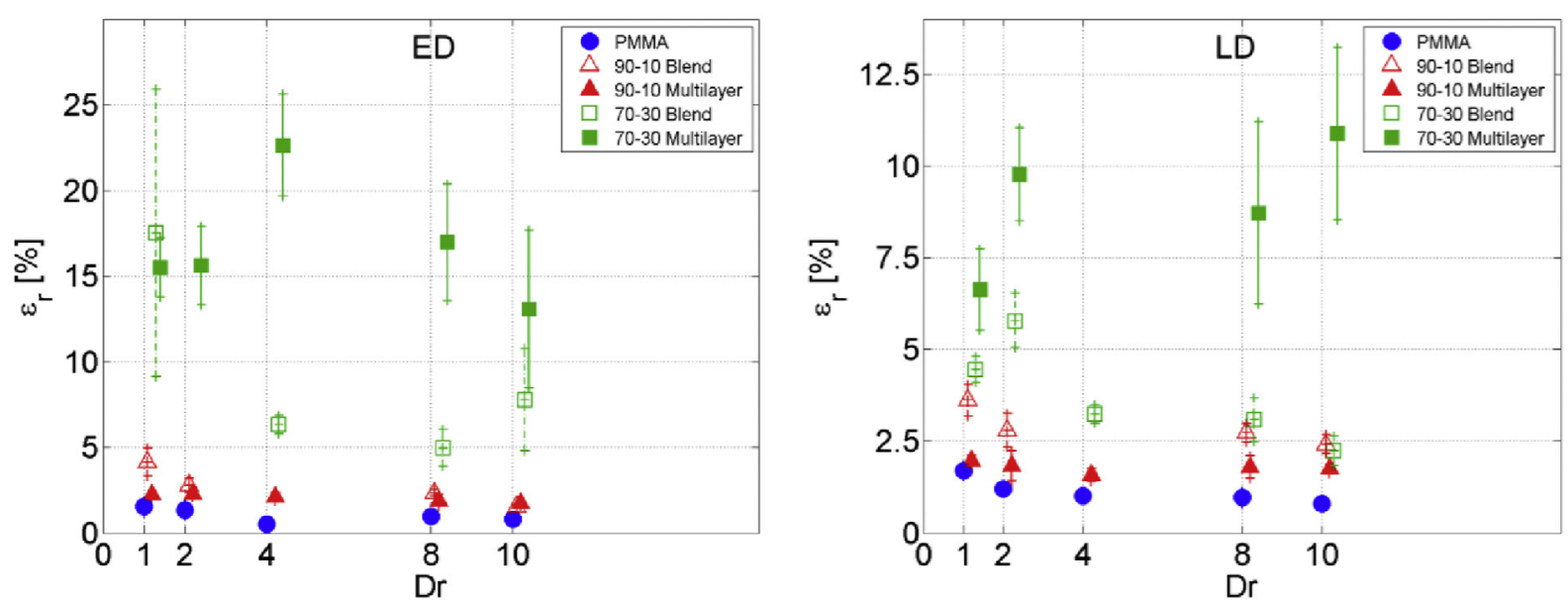

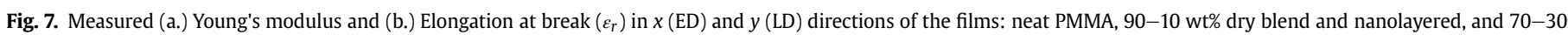
wt\% dry blend and nanolayered, at different draw ratios (Dr).

a.

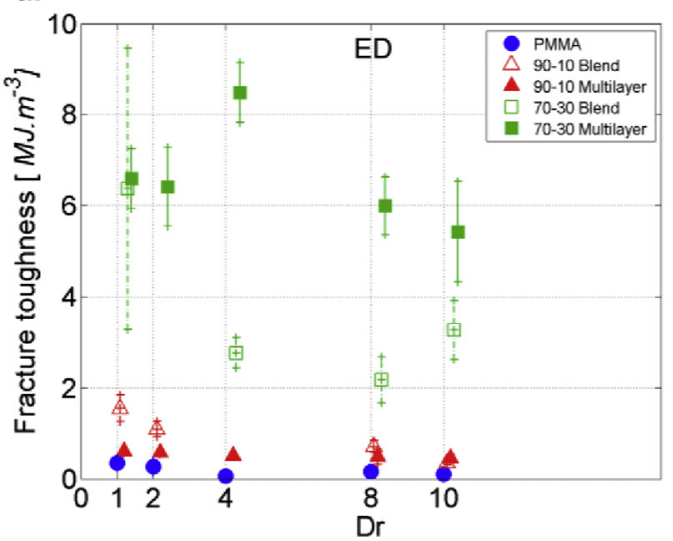

b.

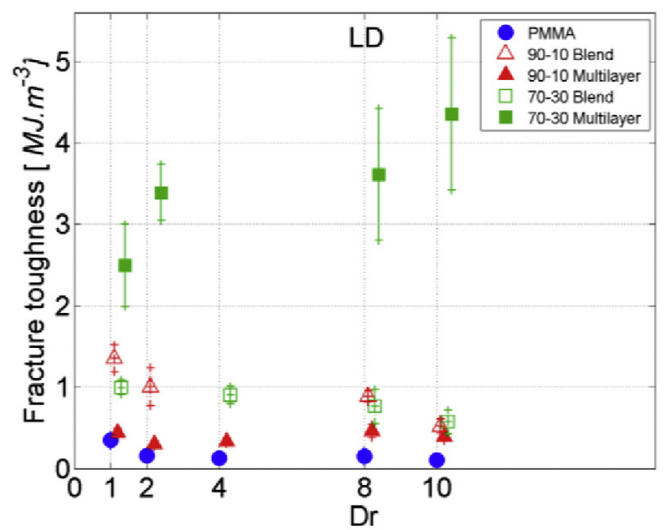

Fig. 8. Fracture toughness as function of draw ratio for the films in ED (a.) and LD (b.): reference PMMA, $90-10$ wt\% dry blend and nanolayered, and $70-30$ wt\% dry blend and nanolayered.

phase in the multilayer films. However, in this study no reference values (for PS or classically blended films) were provided [32].

Then, a fracture toughness increase with decreasing thicknesses of the layers is not observed in the extrusion direction for the PMMA/MAM ( $D r=10$ corresponding to nominal thicknesses of 70 $\mathrm{nm}$ and $\mathrm{Dr}=1$ to thicknesses of $\sim 500 \mathrm{~nm}$, see Table 1 ). It is possible 
that in these systems, the number of layers (i.e. the number of interfaces) rather than their individual thickness is the relevant parameter for the observed reinforcement. More surprisingly, the fracture toughness measured for $70-30 \mathrm{wt} \%$ blends in the lateral direction does not display much lower values than in the extrusion direction $\left(\sim 3 \mathrm{MJ} / \mathrm{m}^{3}\right.$ compared to $\left.\sim 6 \mathrm{MJ} / \mathrm{m}^{3}\right)$, contrary to what was observed by Korley (as high as an order of magnitude difference for films with thinner layers), while the block morphology in PS/SEPS multilayer films was also identified as cylindrical [32]. Moreover, in the PMMA/MAM films, the increase in toughness when compared to dry-blend films is even much pronounced in the lateral direction than in the extrusion direction, as mentioned previously. As of now, a deep understanding of these observed differences in behaviors for somewhat similar multilayer systems is still lacking, and a more detailed comparison of the rupture mechanisms (crazes, shear bands ...) would be needed.

\section{Conclusion}

In this study, the effect of a double level of structuration in a blend of a glassy matrix (PMMA) reinforced by a copolymer triblock (MAM) with a rubbery middle-block on the mechanical response of the material was investigated. This double level in the material architecture is obtained using an innovative process, multilayer coextrusion. The obtained material is then made of alternating nanometric layers of MAM and PMMA, reminiscent of a laminated glass, and within the MAM layers, MAM self-assembles into a welldefined morphology identified as cylindrical, with the aim of reinforcing the PMMA with the MAM rubbery phase. These materials have been compared to neat PMMA films and classically coextruded PMMA/MAM blends (single-screw, dry-blend) where the blend morphology is salami-like (nanoinclusions of PMMA into micron-sized MAM nodules dispersed in the PMMA matrix, without control on the size, dispersion, and distribution of the nodules). The effects of composition and of processing parameters (draw ratio) have also been studied.

The main results can be summarized as followed:

The 70-30 wt\% (16.1 wt\% soft phase) PMMA reinforced by MAM multilayer films show a better mechanical response in terms of fracture toughness than unreinforced PMMA in both directions (by roughly a factor of 20 ), but also than the dry blend films. The measured reinforcement is significantly higher (by a factor of 2-4) compared to those films, independently of the solicitation direction. This improvement in the mechanical response may be attributed to the differences in the nanostructures obtained with the two different processes. In the multilayer structure, a double level of organization is achieved: thousands of continuous alternating (PMMA and MAM) nanolayers, and within the MAM layer a cylindrical arrangement of the BCP. On the contrary, no control of the morphology, of the blend and of the BCP, can be achieved with classically extruded blends. To gain a better understanding in the correlation between nanostructures and mechanical properties, mechanical testing at higher speeds (impact resistance) and in-situ SAXS experiment during sample deformation may be envisioned.

Still, this study opens the way for the design of "nano-laminated organic glass". These nanolayered materials with well-organized nanostructures within the layers, obtained via an industrially scalable one-step process show promises for applications where such organic glasses with high impact resistance are needed.

\section{Acknowledgements}

Arkema is acknowledged for providing the triblock copolymer and advices concerning the staining. TEM observations were conducted in the electron microscopy facility of Institut de Biologie
Paris-Seine (IBPS/FR3631 - UPMC/CNRS - Paris, France), with the help of G. Frebourg. We thank the SOLEIL synchrotron and the SWING beamline for access to the instrumentation (project $\mathrm{n}^{\circ}$ 20160438). More particularly, we are grateful to J. Perez (beamline manager) and T. Bizien (beamline scientist) for the help to obtain the SAXS data. We would like to acknowledge A. Guinault and A. Grandmontagne for their help with the nanolayer coextrusion setup, A. Gaudy for the mechanical tests, and C. Sollogoub, S. Bourrigaud, S. Tencé-Girault, C. Sinturel and L.Rubatat for fruitful discussions throughout the project.

\section{Appendix A. Supplementary data}

Supplementary data related to this article can be found at https://doi.org/10.1016/j.polymer.2018.06.048.

\section{References}

[1] C.B. Bucknall, Toughened Plastics, Springer Netherlands, Dordrecht, 1977.

[2] A.M. Donald, E.J. Kramer, The competition between shear deformation and crazing in glassy polymers, J. Mater. Sci. 17 (7) (1982) 1871-1879.

[3] B. Bilé, J.M. Gloaguen, J.M. Lefebvre, J.P. Tancrez, Fracture behaviour of high impact polystyrene influence of particle morphology, Plast. Rubber Compos. 30 (2) (2001) 77-81.

[4] C. Wrotecki, P. Heim, P. Gaillard, Rubber toughening of poly(methyl methacrylate). Part I: effect of the size and hard layer composition of the rubber particles, Polym. Eng. Sci. 31 (4) (1991) 213-217.

[5] C. Wrotecki, P. Heim, P. Gaillard, Rubber toughening of poly(methyl methacrylate). Part II: effect of a twin population of particle size, Polym. Eng. Sci. 31 (4) (1991) 218-222

[6] C.J. Hooley, D.R. Moore, M. Whale, M.J. Williams, Fracture toughness of rubber modified PMMA, Plast. Rubber Process. Appl. 1 (1981) 345-349.

[7] J.M. Gloaguen, P. Steer, P. Gaillard, C. Wrotecki, J.M. Lefebvre, Plasticity and fracture initiation in rubber-toughened poly(methyl methacrylate), Polym. Eng. Sci. 33 (12) (1993) 748-753.

[8] J.M. Gloaguen, J.M. Lefebvre, C. Wrotecki, Critical energy for crack initiation in rubber-toughened poly(methyl methacrylate), Polymer 34 (2) (1993) 443-445.

[9] P. Heim, C. Wrotecki, M. Avenel, P. Gaillard, High impact cast sheets of pol$\mathrm{y}$ (methyl methacrylate) with low levels of polyurethane, Polymer 34 (8) (1993) 1653-1660.

[10] C.J.G. Plummer, P. Béguelin, H.H. Kausch, Microdeformation in core-shell particle modified polymethylmethacrylates, Colloids Surf. Physicochem. Eng. Asp. 153 (1-3) (1999) 551-566.

[11] L. Lalande, C.J.G. Plummer, J.A.E. Månson, P. Gérard, The influence of matrix modification on fracture mechanisms in rubber toughened polymethylmethacrylate, Polymer 47 (7) (2006) 2389-2401.

[12] L Lalande, C.J.G. Plummer, J.A.E. Månson, P. Gérard, Microdeformation mechanisms in rubber toughened PMMA and PMMA-based copolymers, Eng. Fract. Mech. 73 (16) (2006) 2413-2426.

[13] Design and applications of nanostructured polymer blends and nanocomposite systems, in: S. Thomas, R. Shanks, S. Chandrasekharakurup (Eds.) Micro and Nano Technologies, William Andrew Publishing, Boston, 2015.

[14] J.D. Tong, R. Jerôme, Synthesis of poly(methyl methacrylate)-b-poly(n-butyl acrylate)-b-poly(methyl methacrylate) triblocks and their potential as thermoplastic elastomers, Polymer 41 (7) (2000) 2499-2510.

[15] G. Moineau, M. Minet, P. Dubois, P. Teyssié, T. Senninger, R. Jérôme, Controlled radical polymerization of (Meth)Acrylates by ATRP with $\mathrm{NiBr} 2(\mathrm{PPh} 3) 2$ as catalyst, Macromolecules 32 (1) (1999) 27-35.

[16] J.D. Tong, G. Moineau, P. Leclère, Brédas, R. Lazzaroni, R. Jérôme, Synthesis morphology, and mechanical properties of poly(methyl methacrylate)-bpoly(n-butyl acrylate)-b-poly(methyl methacrylate) triblocks. Ligated anionic polymerization vs atom transfer radical polymerization, Macromolecules 33 (2) (2000) 470-479.

[17] L. Lalande, Structure et Mécanismes de Microdéformation de Polymethylmethacrylates Renforcés Au Choc, École polytechnique fédérale de Lausanne, EPFL, Lausanne, 2007.

[18] A.-V. Ruzette, S. Tencé-Girault, L. Leibler, F. Chauvin, D. Bertin, O. Guerret P. Gérard, Molecular disorder and mesoscopic order in polydisperse acrylic block copolymers prepared by controlled radical polymerization, Macromolecules 39 (17) (2006) 5804-5814.

[19] J. Pinto, M. Dumon, M. Pedros, J. Reglero, M.A. Rodriguez-Perez, Nanocellular CO2 foaming of PMMA assisted by block copolymer nanostructuration, Chem. Eng. J. 243 (Supplement C) (2014) 428-435

[20] J. Pinto, M. Dumon, M.A. Rodriguez-Perez, R. Garcia, C. Dietz, Block copolymers self-assembly allows obtaining tunable micro or nanoporous membranes or depth filters based on PMMA; fabrication method and nanostructures, J. Phys. Chem. C 118 (9) (2014) 4656-4663.

[21] I.W. Hamley, K.A. Koppi, J.H. Rosedale, F.S. Bates, K. Almdal, K. Mortensen, 
Hexagonal mesophases between lamellae and cylinders in a diblock copolymer melt, Macromolecules 26 (22) (1993) 5959-5970.

[22] F.S. Bates, K.A. Koppi, M. Tirrell, K. Almdal, K. Mortensen, Influence of shear on the hexagonal-to-disorder transition in a diblock copolymer melt, Macromolecules 27 (20) (1994) 5934-5936.

[23] T. Kotaka, T. Miki, K. Arai, Morphology-mechanical property relationship of a polystyrene-polybutadiene-polystyrene block copolymer and its blends with homopolymers, J. Macromol. Sci. Part B 17 (2) (1980) 303-336.

[24] D. Wang, K. Nakajima, S. Fujinami, Y. Shibasaki, J-O. Wang, T. Nishi, Charac terization of morphology and mechanical properties of block copolymers using atomic force microscopy: effects of processing conditions, Polymer 53 (9) (2012) 1960-1965.

[25] Chapter 3: Nanostructures of two-component amorphous block copolymers: effect of chain architecture, in: G. Michler, F. Baltá-Calleja (Eds.), Mechanical Properties of Polymers Based on Nanostructure and Morphology, CRC Press 2005, pp. 115-136.

[26] Chapter 10: Micromechanical mechanisms of toughness enhancement in nanostructured amorphous and semicrystalline polymers, in: G. Michler F. Baltá-Calleja (Eds.), Mechanical Properties of Polymers Based on Nanostructure and Morphology, CRC Press, 2005, pp. 419-428.

[27] C.C. Honeker, E.L. Thomas, Impact of morphological orientation in determining mechanical properties in triblock copolymer systems, Chem. Mater. 8 (8) (1996) 1702-1714.

[28] I. Yamaoka, M. Kimura, Effects of morphology on mechanical properties of a
SBS triblock copolymer, Polymer 34 (21) (1993) 4399-4409.

[29] C. Ye, G. Singh, M.L. Wadley, A. Karim, K.A. Cavicchi, B.D. Vogt, Anisotropic mechanical properties of aligned polystyrene-block-polydimethylsiloxane thin films, Macromolecules 46 (21) (2013) 8608-8615.

[30] M. Ponting, A. Hiltner, E. Baer, Polymer nanostructures by forced assembly: process, structure, and properties, Macromol. Symp. 294 (1) (2010) 19-32.

[31] T.M. Burt, J. Keum, A. Hiltner, E. Baer, L.T.J. Korley, Confinement of elastomeric block copolymers via forced assembly coextrusion, ACS Appl. Mater. Interfaces 3 (12) (2011) 4804-4811.

[32] T.M. Burt, A.M. Jordan, L.T.J. Korley, Toward anisotropic materials via forced assembly coextrusion, ACS Appl. Mater. Interfaces 4 (10) (2012) 5155-5161.

[33] S. Roland, G. Miquelard-Garnier, M. Gervais, A. Guinault, C. Sollogoub, Controlling the order of triblock copolymer via confinement induced by forced self-assembly, Mater. Today Commun 6 (2016) 37-43.

[34] J.-S. Montana, S. Roland, E. Richaud, G. Miquelard-Garnier, From equilibrium lamellae to out-of-equilibrium cylinders in triblock copolymer nanolayers obtained via multilayer coextrusion, Polymer 136 (2018) 27-36.

[35] G. David, J. Pérez, Combined sampler robot and high-performance liquid chromatography: a fully automated system for biological small-angle x-ray scattering experiments at the synchrotron SOLEIL SWING beamline, J. Appl. Crystallogr. 42 (5) (2009) 892-900.

[36] A. Bironeau, J. Dirrenberger, C. Sollogoub, G. Miquelard-Garnier, S. Roland, Evaluation of morphological representative sample sizes for nanolayered polymer blends, J. Microsc. 264 (1) (2016) 48-58. 